\title{
Numerical Computation and Investigation of the Characteristics of Microscale Synthetic Jets
}

\author{
Ann Lee, ${ }^{1}$ Guan H. Yeoh, ${ }^{1,2}$ Victoria Timchenko, ${ }^{1}$ and John Reizes $^{1}$ \\ ${ }^{1}$ School of Mechanical \& Manufacturing Engineering, University of New South Wales, Sydney, NSW 2052, Australia \\ ${ }^{2}$ Australian Nuclear Science and Technology Organisation (ANSTO), PMB 1, Menai, NSW 2234, Australia \\ Correspondence should be addressed to Guan H. Yeoh, guan.yeoh@ansto.gov.au
}

Received 12 December 2010; Accepted 2 February 2011

Academic Editor: Chaoqun Liu

Copyright (C 2011 Ann Lee et al. This is an open access article distributed under the Creative Commons Attribution License, which permits unrestricted use, distribution, and reproduction in any medium, provided the original work is properly cited.

\begin{abstract}
A synthetic jet results from periodic oscillations of a membrane in a cavity. Jet is formed when fluid is alternately sucked into and ejected from a small cavity by the motion of membrane bounding the cavity. A novel moving mesh algorithm to simulate the formation of jet is presented. The governing equations are transformed into the curvilinear coordinate system in which the grid velocities evaluated are then fed into the computation of the flow in the cavity domain thus allowing the conservation equations of mass and momentum to be solved within the stationary computational domain. Numerical solution generated using this moving mesh approach is compared with an experimental result measuring the instantaneous velocity fields obtained by $\mu$ PIV measurements in the vicinity of synthetic jet orifice $241 \mu \mathrm{m}$ in diameter issuing into confined geometry. Comparisons between experimental and numerical results on the streamwise component of velocity profiles at the orifice exit and along the centerline of the pulsating jet in microchannel as well as the location of vortex core indicate that there is good agreement, thereby demonstrating that the moving mesh algorithm developed is valid.
\end{abstract}

\section{Introduction}

A synthetic jet, similar to a pulsed jet, is formed from the ingestion and expulsion of fluid through an orifice into a fluid-filled space [1]. One unique feature is that the jet is produced entirely by the working fluid of the flow system. It can thus transfer linear momentum to the flow system without net mass injection across the flow boundary. The mechanism of such a jet is sometimes known as a "zero-net-mass-flux" jet [2]. In a synthetic jet, the actuating flow is generated by an oscillating diaphragm located opposite the orifice in a cavity. As the diaphragm oscillates, the flow exhibits a periodic behavior. Under certain operating conditions, the fluid separates, leading to the formation of discrete vortical structures. This so-called vortex shedding phenomenon is important for thermal management applications because the cooling performance is highly dependent on the interaction of these trains of discrete vortical structures and the wall to be cooled.

A number of useful studies have been carried out on synthetic jets in the context of pulsating jet actuators impinging on submerged surfaces in quiescent fluid media in order to better understand the thermal characteristics for localized cooling. Campbell et al. [3] illustrated the use of synthetic air micro jets for effective cooling of laptop processors while Mahalingam et al. $[4,5]$ established the feasible application of synthetic jets for high-power electronic cooling through an integrated active heat sink. Smith and Swift [6] as well as Pavlova and Amitay [7] indicated that heat transfer enhancement increased dramatically through the use of synthetic jets over continuous jets thereby offering better cooling performance. Gillespie et al. [8] investigated the effectiveness of a rectangular synthetic jet impinging on an unconfined heated plate exposed to the ambient, while Trávníček and Tesař $[9,10]$ proposed the alternative use of annular synthetic jet which has shown to provide more attractive features over a simple round nozzle impinging jet $[11,12]$, in particular the increased wall transport phenomena by the applied pulsation.

To account for the transient movement of the actuator wall, an explicit tracking of the membrane accompanied by a moving mesh is implemented via solving the governing 
equations in a form represented by a three-dimensional body-fitted curvilinear coordinate system in a fixed computational domain. A methodology for moving the mesh points, which respects the boundary movement(s), eliminates the need for remeshing, and satisfies the geometric conservation law (GCL), is adopted. The published literature reports very few numerical analyses on the interactive combination of the highly favorable synthetic jet characteristics and proven effectiveness of microchannel flows. At the same time, very limited experimental data is available for comparison. The objective of this paper is to assess the validity and performance of the grid movement methodology against micro-scale synthetic jet experiment carried out at the University of New South Wales.

\section{Mathematical Model}

In this section, the governing equations and the numerical methodology used for the simulation of moving boundary flows are presented. A collocated, structured, and body-fitted grid is employed. The physical coordinates $(x, y, z$, and $t)$ are related to the computational coordinates $(\varepsilon, \gamma, \omega$, and $\tau)$ through the generic transformations:

$$
\begin{gathered}
x=x(\varepsilon, \gamma, \omega, t), \\
y=y(\varepsilon, \gamma, \omega, t), \\
z=z(\varepsilon, \gamma, \omega, t), \\
\quad t=\tau .
\end{gathered}
$$

Initially at rest $(t=0)$, the grid within the physical domain can be constructed via the method of transfinite interpolation according to Gordon and Thiel [13] which consists of generating the interior mesh from the boundary grid data using appropriate interpolation functions or "blending" functions. In three dimensions, the interpolation operation is

$$
\vec{r}_{i, j, k}=P^{i}+P^{j}+P^{k}-P^{i} P^{j}-P^{i} P^{k}-P^{j} P^{k}+P^{i} P^{j} P^{k},
$$

where $\vec{r} \equiv r^{i} \equiv(\mathrm{x}, \mathrm{y}, \mathrm{z})$,

$$
\begin{aligned}
P^{i}= & f_{i} \vec{r}_{1, j, k}+\left(1-f_{i}\right) \vec{r}_{I, j, k}, \\
P^{j}= & g_{j} \vec{r}_{j, 1, k}+\left(1-g_{j}\right) \vec{r}_{i, j, k}, \\
P^{k}= & h_{k} \vec{r}_{i, j, 1}+\left(1-h_{k}\right) \vec{r}_{i, j, k}, \\
P^{i} P^{j}= & f_{i} g_{j} \vec{r}_{I, J, k}+f_{i}\left(1-g_{j}\right) \vec{r}_{I, 1, k}+\left(1-f_{i}\right) g_{j} \vec{r}_{1, J, k} \\
& +\left(1-f_{i}\right)\left(1-g_{j}\right) \vec{r}_{1,1, k}, \\
P^{i} P^{k}= & f_{i} h_{k} \vec{r}_{I, j, K}+f_{i}\left(1-h_{k}\right) \vec{r}_{I, j, 1}+\left(1-f_{i}\right) h_{k} \vec{r}_{1, j, K} \\
& +\left(1-f_{i}\right)\left(1-h_{k}\right) \vec{r}_{1, j, 1}, \\
P^{j} P^{k}= & g_{j} h_{k} \vec{r}_{i, J, k}+g_{j}\left(1-h_{k}\right) \vec{r}_{i, J, 1}+\left(1-g_{j}\right) h_{k} \vec{r}_{i, 1, K} \\
& +\left(1-g_{j}\right)\left(1-h_{k}\right) \vec{r}_{i, 1,1},
\end{aligned}
$$

$$
\begin{aligned}
P^{i} P^{j} P^{k}= & f_{i} g_{j} h_{k} \vec{r}_{I, J, K}+f_{i} g_{j}\left(1-h_{k}\right) \vec{r}_{I, J, 1} \\
& +f_{i}\left(1-g_{j}\right) h_{k} \vec{r}_{I, 1, K}+f_{i}\left(1-g_{j}\right)\left(1-h_{k}\right) \vec{r}_{I, 1,1} \\
& +\left(1-f_{i}\right) g_{j} h_{k} \vec{r}_{1, J, K}+\left(1-f_{i}\right) g_{j}\left(1-h_{k}\right) \vec{r}_{1, J, 1} \\
& +\left(1-f_{i}\right)\left(1-g_{j}\right) h_{k} \vec{r}_{1,1, K} \\
& +\left(1-f_{i}\right)\left(1-g_{j}\right)\left(1-h_{k}\right) \vec{r}_{1,1,1} .
\end{aligned}
$$

In the above equation, $f_{i}$ varies monotonically from $f_{1}=$ 0 to $f_{I}=1$ for $i=1,2, \ldots, I$. Analogous expressions involving functions $g_{j}$ and $h_{k}$ apply for interpolation in the $j$ and $k$ directions for $j=1,2, \ldots, J$ and $k=1,2, \ldots, K$, respectively. For simplicity, the blending functions $f_{i}, g_{j}$, and $h_{k}$ may be taken to be linear. They may also be formulated with added complexity to concentrate more grid points near the physical boundaries or to include the specification of derivative boundary conditions to force the grid lines to intersect the physical boundaries orthogonally.

2.1. Governing Equations. The general form of the governing equations (continuity, momentum, enthalpy, and solid temperature) transformed into the body-fitted curvilinear coordinate system $\left(\xi^{l} \equiv(\varepsilon, \gamma, \omega)\right)$ is given accordingly as follows.

Continuity:

$$
\frac{\partial \rho_{l}}{\partial \tau}+\frac{1}{\sqrt{g}}\left[\frac{\partial}{\partial \xi^{l}}\left(\rho_{l}\left(U^{\xi^{l}}-U_{g}^{\xi^{l}}\right)\right)\right]=0 .
$$

Momentum:

$$
\begin{aligned}
\frac{\partial\left(\rho_{l} U_{i}\right)}{\partial \tau} & +\frac{1}{\sqrt{g}}\left[\frac{\partial}{\partial \xi^{l}}\left(\rho_{l}\left(U^{\xi^{l}}-U_{g}^{\xi^{l}}\right) U_{i}\right)\right] \\
= & -\frac{1}{\sqrt{g}} \frac{\partial}{\partial \xi^{l}}\left(\beta_{i l} p\right)+\frac{1}{\sqrt{g}} \frac{\partial}{\partial \xi^{l}}\left(\mu_{l} \sqrt{g} g^{l m} \frac{\partial U_{i}}{\partial \xi^{m}}\right) \\
& +\frac{1}{\sqrt{g}} \frac{\partial}{\partial \xi^{l}}\left(\mu_{l} \frac{\beta_{k l}}{\sqrt{g}} \frac{\partial}{\partial \xi^{m}}\left(\beta_{l m} U_{k}\right)\right)
\end{aligned}
$$

where $\rho_{l}$ and $\mu_{l}$ are the density and dynamic viscosity, respectively.

The various coefficients in (6) and (7) are

$$
\beta_{i l}=\frac{\partial r^{j}}{\partial \xi^{m}} \frac{\partial r^{k}}{\partial \xi^{n}}-\frac{\partial r^{k}}{\partial \xi^{n}} \frac{\partial r^{j}}{\partial \xi^{m}}
$$

with $i, j, k$ and $l, m, n$ being cyclic:

$$
\sqrt{g} g^{l m}=\sum_{k=1}^{3} \frac{\beta_{k l} \beta_{k m}}{\sqrt{g}} \quad \sqrt{g}=\operatorname{det}\left|\begin{array}{lll}
\frac{\partial x}{\partial \varepsilon} & \frac{\partial y}{\partial \varepsilon} & \frac{\partial z}{\partial \varepsilon} \\
\frac{\partial x}{\partial \gamma} & \frac{\partial y}{\partial \gamma} & \frac{\partial z}{\partial \gamma} \\
\frac{\partial x}{\partial \omega} & \frac{\partial y}{\partial \omega} & \frac{\partial z}{\partial \omega}
\end{array}\right| .
$$


The fluid velocity components in the $\varepsilon, \gamma$, and $\omega$ directions are given as

$$
U^{\xi^{l}}=\sum_{k=1}^{3} \beta_{k l} U_{k}
$$

while the grid velocity components in the $\varepsilon, \gamma$, and $\omega$ directions are, respectively, are given as

$$
U_{g}^{\xi^{l}}=\sum_{k=1}^{3} \beta_{k l} U_{g}^{l}
$$

In (13), $U_{g}^{l}$ are the instantaneous grid velocities in $x$, $y$, and $z$ directions at a fixed point $(\varepsilon, \gamma$, and $\omega)$ in the computational domain. These are given as

$$
U_{g}^{1}=\left(\frac{\partial x}{\partial t}\right)_{\varepsilon, \gamma, \omega}, \quad U_{g}^{2}=\left(\frac{\partial y}{\partial t}\right)_{\varepsilon, \gamma, \omega}, \quad U_{g}^{3}=\left(\frac{\partial z}{\partial t}\right)_{\varepsilon, \gamma, \omega}
$$

The grid movement methodology that is employed in this present study to determine the instantaneous grid velocities in (14) is described in the next section.

2.2. Grid Movement Methodology. The use of body-fitted coordinate system along with the time-dependent transformation approach eliminates the need to explicitly track the membrane in the physical domain. Furthermore, the discretisation accuracy near the moving membrane is far better resolved as the grid lines conform to the body shape. One key feature of the grid movement methodology in this paper is the avoidance of the time-consuming remeshing step of the grid within the physical domain. The body-fitted moving mesh algorithm based on the approach proposed by Anwer et al. [14] is applied herein. It is a cheaper and accurate alternative for moderately complex geometries, and, more importantly, it automatically satisfies the GCL in which failure to enforce the GCL leads to inaccuracies in the computations.

Figure 1 shows a typical domain of the synthetic jet generator whereby the moving membrane is normally located at the bottom of the cavity. The surface mesh points at the top of the cavity can be taken to be fixed while the surface mesh points surrounding the moving membrane are allowed to move according to the imposed instantaneous displacement function.

At the bottom surface, the instantaneous grid velocities can be analytically obtained through differentiating the instantaneous displacement function with time. In order to determine the internal instantaneous grid velocities, the velocities over the mesh points within the region can be gradually relaxed from their corresponding rigid values at the top surface through a weighting function $f(d)$, where $\mathrm{d}$ is the distance of the mesh point under consideration from the origin of the frame attached to the surface of the moving

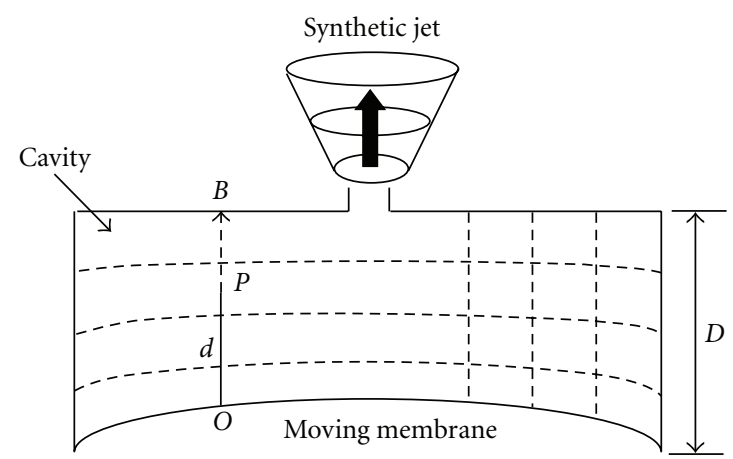

Figure 1: A typical geometric representation of the synthetic jet generator.

membrane. Consider a mesh point $P$ as shown in Figure 1 . Denoting the distance of $B$ from the dynamic origin $O$ as $R$, the mesh velocity at point $P$ is given as

$$
U_{g}^{l}(P)=U_{g, \text { mambrane }}^{l}(P)(1-f(d))
$$

where $f(d)=0$ for $d=d_{\min }$ and $f(d)=1$ for $d=D$. The weighting function $f(d)$ may take any suitable form. For simplicity, a linear weighting function is adopted: $(d-$ $\left.d_{\min }\right) /\left(D-d_{\min }\right)$. As aforementioned, the membrane velocity $U_{g \text {,mambrane }}^{l}(P)$ in $(15)$ can be determined from an analytical function, to be described later. Once the mesh velocities are obtained via (15), the mesh point locations at the new time are found by

$$
\begin{aligned}
& x^{n+1}=x^{n}+U_{g}^{1}(P) \Delta t, \\
& y^{n+1}=y^{n}+U_{g}^{2}(P) \Delta t, \\
& z^{n+1}=z^{n}+U_{g}^{3}(P) \Delta t .
\end{aligned}
$$

2.3. Numerical Scheme. The governing equations are discretised by the finite volume technique (FVM) on a structured collocated grid arrangement. In the interior of the flow domain, the hybrid differencing scheme which switches between the first-order upwind differencing and secondorder central differencing based on the local Péclet number is adopted for the advection terms. The pressure gradients and diffusion terms are discretised using the second-order central differencing scheme. Pressure correction algorithm such as SIMPLE is applied to link the collocated velocity and pressure fields. The determination of the velocities at the control volume faces is achieved through the application of the Rhie and Chow [15] interpolation procedure. A full-implicit iterative procedure is employed to capture the unsteady physics of the flow. The algebraic forms of the governing equations are solved by employing the strongly implicit procedure (SIP) by Stone [16]. Within each time step, convergence is ascertained when the mass residual falls below $10^{-5}$. 


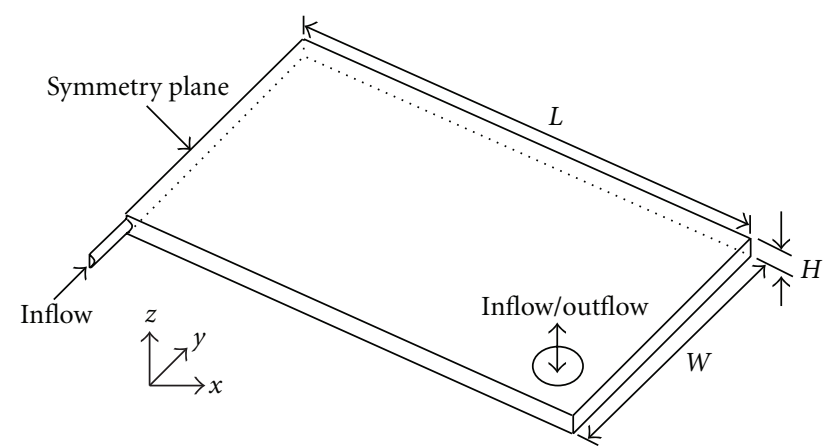

FIgURE 2: Schematic diagram of three-dimensional micro synthetic jet.

\section{Problem Definition}

A schematic diagram of the three-dimensional geometry is shown in Figure 2. The width and depth of the rectangular test section have been designed to be of $5000 \mu \mathrm{m}$ and $500 \mu \mathrm{m}$, respectively. To enhance the numerical calculations, the length of rectangular test section was halved, $15 \mathrm{~mm}$ long, using the assumption of symmetry relative to the centerline of the jet. To model the syringe which acted as the piston/cylinder device for the synthetic jet actuator and was connected at the mid-depth of the test section, an additional pipe with a diameter of $700 \mu \mathrm{m}$ was attached to the computational domain to be able to generate the pulsating flow through the orifice. In order to simulate the piston effect as the plunger moved in and out of the barrel of the syringe, the length of this pipe was varied through time.

For the geometry described in Figure 2, a mesh of 95 $\times 36 \times 41$ was generated which resulted in a total of 140,220 control volumes. Denser mesh was concentrated around the orifice to resolve the fluid flow characteristics of the synthetic jet. Sensitivity analysis was performed by comparing the numerical results against a finer mesh (twice the number of grid points); the variation of the fluid flow fields was found to be negligible. A time-step of 1/200 of the period of oscillation of the membrane was employed. For the inlet/outlet boundary located some distance away from the orifice, a static pressure was imposed to allow the possibility of the fluid entering or leaving the domain.

The motion of the synthetic jet actuator was fully simulated in the calculation. Structural simulations indicated that the plunger could be simulated by varying in a sinusoidal fashion in time, so that, rather than solving the complex fluid-solid interaction problem, the instantaneous displacement of the membrane $Y_{m}$ was assumed to be

$$
Y_{m}=A \sin (2 \pi f t)
$$

where $A$ is the displacement amplitude and $f$ is the frequency of oscillation. The membrane oscillation frequency and amplitude are activated based on the frequency $f=240 \mathrm{~Hz}$ and amplitude $A=265 \mu \mathrm{m}$.

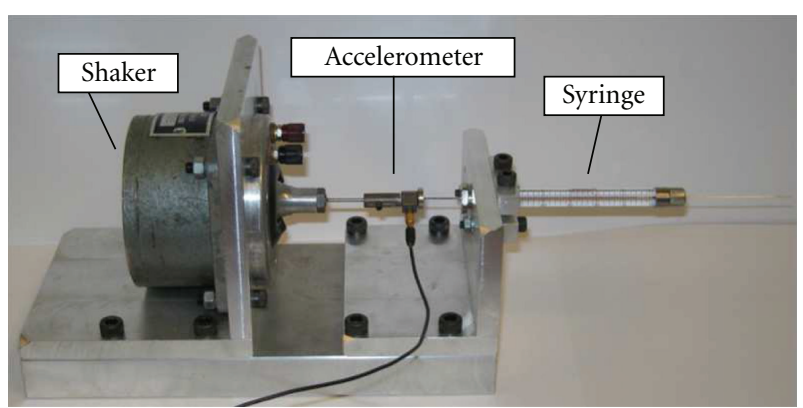

Figure 3: Experimental apparatus.

\section{Experimental Setup}

The validity and performance of the grid movement methodology is assessed against experiments of micro synthetic jets in quiescent fluid media conducted by Sinclair et al. [17] at the University of New South Wales. In their experiment, a $25 \mu \mathrm{L}$ syringe acting as a piston/cylinder device for the synthetic jet actuator that consists of a $700 \mu \mathrm{m}$ diameter plunger in a $730 \mu \mathrm{m}$ diameter barrel is used. The barrel of the syringe is connected to a $50 \mathrm{~mm}$ long, 25-gauge stainless steel needle (ID/OD $=241 / 500 \mu \mathrm{m}$ ) which is connected to the test section and acts as the orifice for the synthetic jet. The plunger is connected to a $1.1 \mathrm{~kg}$ force voice coil shaker by a steel connecting rod and grub screw connection piece. An ICP accelerometer is fixed with adhesion between the connection piece and the plunger. As can be seen in Figure 3, the shaker and syringe configuration were held in line together by a rig made from aluminum.

A sinusoidal waveform produced by function generator and amplified by a $25 \mathrm{~W}$ amplifier was supplied to the shaker, to oscillate the plunger. The shaker motion was captured by the accelerometer whose factory-calibrated sensitivity was given as $10.87 \mathrm{mV} / \mathrm{m} / \mathrm{s}^{2}$. The sensitivity was confirmed for the current experimental setup by calibrating it against an accelerometer calibrator. The accelerometer was supplied with a constant current signal from a charge amplifier with ICP mode which outputs the signal from the accelerometer to a data acquisition system.

The syringe needle connects to a rectangular test section $5 \mathrm{~mm}$ wide, $30 \mathrm{~mm}$ long, and $500 \mu \mathrm{m}$ deep shown in Figure 4. The needle lies perpendicular to the channel with the orifice in line with one of the test section walls. The working fluid entered the channel through inlet/outlets located behind each end of the rectangular channel. The test section geometry was fabricated in polydimethylsiloxane (PDMS) from a mould consisting of a 25-gauge needle and a $500 \mu \mathrm{m}$ thick aluminum spacer. The PDMS section was bonded to a glass slide with the use of a corona [18]. A 25-gauge needle was inserted into the test section and aligned under a microscope with one of the channel walls. An epoxy was used to secure the needle in position, and silicone sealant was used around the outside edge of the test section and around the inlet/outlet connections for extra durability. 


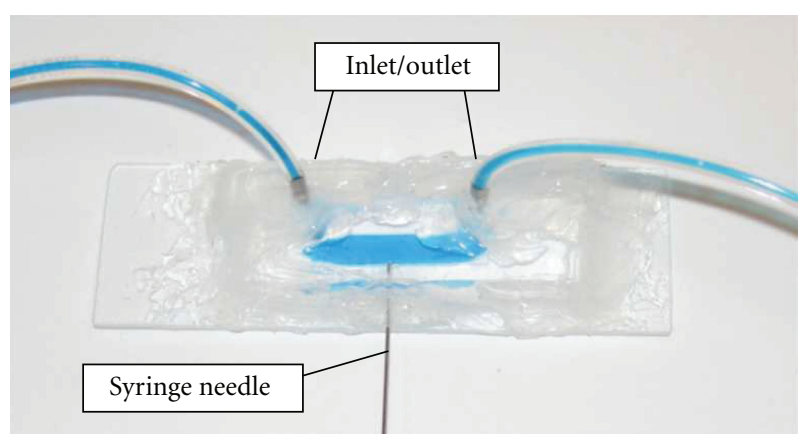

FIGURE 4: The PDMS test section with blue dye added to the water for clarity.

Instantaneous velocity fields were obtained using the $\mu \mathrm{PIV}$ technique with a high-speed $\mu \mathrm{PIV}$ system in a plane bisecting the jet. Image pairs were acquired by a high-speed CMOS camera connected to an inverted microscope. The field of view was volume illuminated by a dual Nd: YLF laser system with a wavelength of $527 \mathrm{~nm}$ and a maximum repetition rate of $10,000 \mathrm{~Hz}$ per laser head. The timing of the laser system and camera was controlled by a PIV synchronizer through the PIVsync software. The flow of purified water was seeded with red fluorescing $2 \mu \mathrm{m}$ diameter. A filter cube allowed the excitation light from the laser to pass through the objective and onto the test section while only allowing the light emitted from the particles to be imaged by the camera. The time delay between pluses for image pairs was $25 \mu \mathrm{s}$ in order to capture the fast moving particles along the centreline.

The spatial resolution of the system is determined by the interrogation process. The final interrogation windows size was $32 \times 32$ pixels $(28 \times 28 \mu \mathrm{m})$, and the depth of correlation was $16 \mu \mathrm{m}$ which defined the interrogation volume. To achieve this resolution a high seeding concentration of $0.09 \%$ was used to ensure 2-3 particles per interrogation volume. With adjacent interrogation windows being overlapped by $50 \%$ the final spatial resolution of the system is $14 \mu \mathrm{m}$. The synchronizing for the high-speed PIV system was based on timing from the high-speed camera. At full camera resolution the maximum rate that image pairs can be acquired at is $500 \mathrm{~Hz}$, which would allow pairs to be taken from 25 different times within the oscillation cycle (for an operating frequency, $f=240 \mathrm{~Hz}$ ).

A multipass interrogation scheme was used with $64 \times$ 64 pixels in the first pass and $32 \times 32$ pixels in the second pass with $50 \%$ overlap used in both passes. The 64 pairs of velocity fields generated at each phase were extracted from a total of 768 cycles and used to produce the 25 phase average velocity fields. All signals generated were captured using a 4-channel 16-bit data acquisition card, which was used to acquire the signal from the accelerometer at a sampling rate of $20 \mathrm{kHz}$. After sampling, the acceleration data was filtered with a series of high-pass filters to remove the DC offset present in the signal without significantly changing the waveform. The data was integrated numerically to determine the velocity profile and integrated again for the displacement profile. More details on the experiment can be referred to in Sinclair et al. [17].

\section{Results and Discussions}

Integration of the accelerometer data yielded a plunger displacement with a profile that appeared qualitatively sinusoidal. A clearance volume between the plunger and syringe wall exists, which could also permit fluid flow. Therefore the exact volume of fluid ejected through the orifice over a cycle could not be calculated from the measured plunger displacement.

The use of a high-speed $\mu$ PIV system, while capable of measuring up to 10,000 image pairs per second, did not allow phase locked measurements to be made, which meant that special care was needed to be taken to ensure accurate measurement. The devised dividing of the actuation cycle into phases required that all signals were to be at the desired frequency and remain constant throughout the measurement period. The data taken from the accelerometer was used to check the actuation frequency over the measurement period of 3.2 seconds or 768 cycles. The actual measured frequency of the actuation cycle in the experiment was $240.01 \mathrm{~Hz}$ which would mean a shift of 0.032 of a cycle by the end of the measurement period. To keep the drift of each phase location below 0.01 of a cycle, only the first 16 image pairs at each phase were used to generate the phase averaged fields. Averaging from only 16 pairs compared to the intended 64 significantly decreased the accuracy of the results.

Without synchronization of the image acquisition to the actuation cycle the locations of each of the 25 measurement points within the cycle are unknown. Thus, the locations of the measurement phases were determined by comparison with the numerical results at a single phase, and all other phases shifted according to their separation. The peak velocity measured from the $\mu \mathrm{PIV}$ data was used as the reference velocity and phase. This maximum velocity was used to determine the maximum velocity that would occur in the simulation, and the phase location of this point in the simulation results was then assigned to the $\mu \mathrm{PIV}$ case.

Nondimensional time $t$ was calculated from the ratio between the physical time and the period of oscillation $t=$ $t^{*} / T$, whilst the distances from the centre line of the jet and also from the orifice are nondimensionalised with respect to diameter of the orifice. Setting $t=0$ at bottom dead center of the plunger stroke, the maximum velocity just outside the orifice in the simulations occurred at $t=0.26$. Therefore the maximum $\mu \mathrm{PIV}$ case was set to $t=0.26$, and all image pairs located were shifted accordingly.

Velocity vector plots for experimental and numerical results for different phases during the first half of the expulsion stage are plotted side by side in Figure 5. In Figure 5(a), the early stage of vortices formation adjacent to the exit of orifice can be seen. At $t=0.18$, the vortices become more visible and shift along with the flow coming out from the orifice in streamwise direction. At the end of 


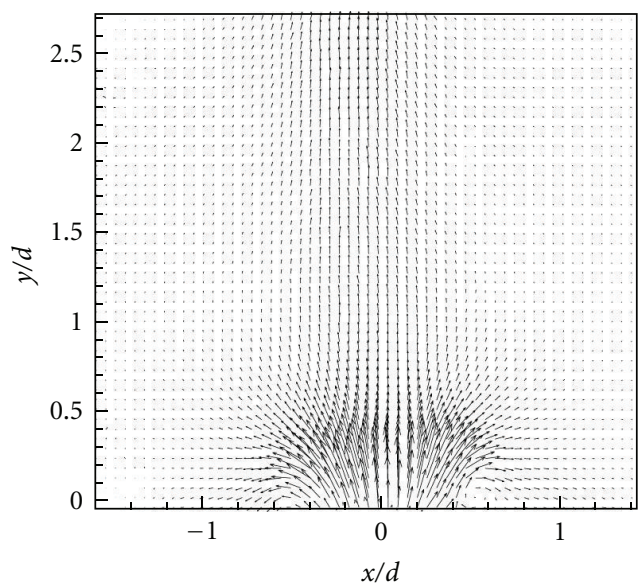

(a)

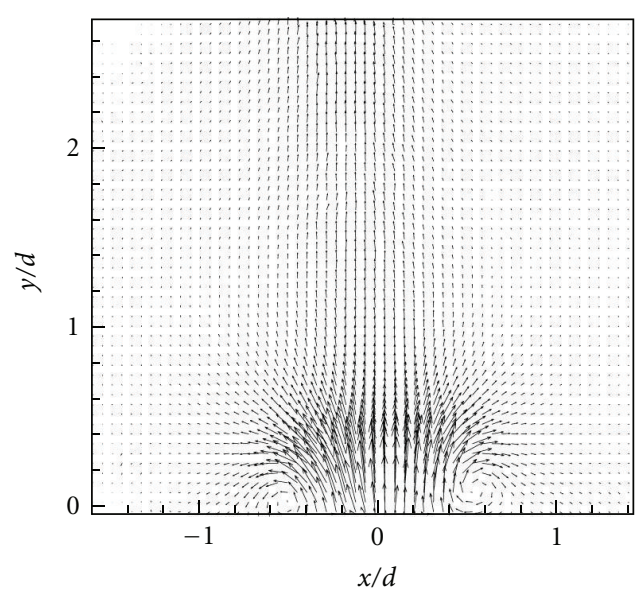

(c)

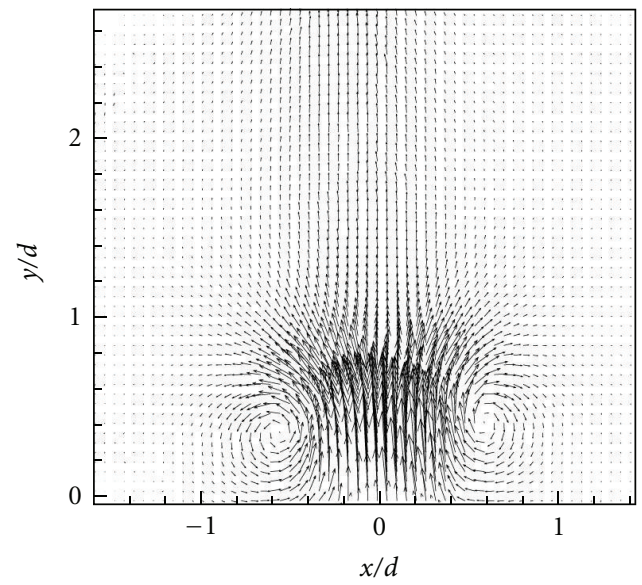

(e)

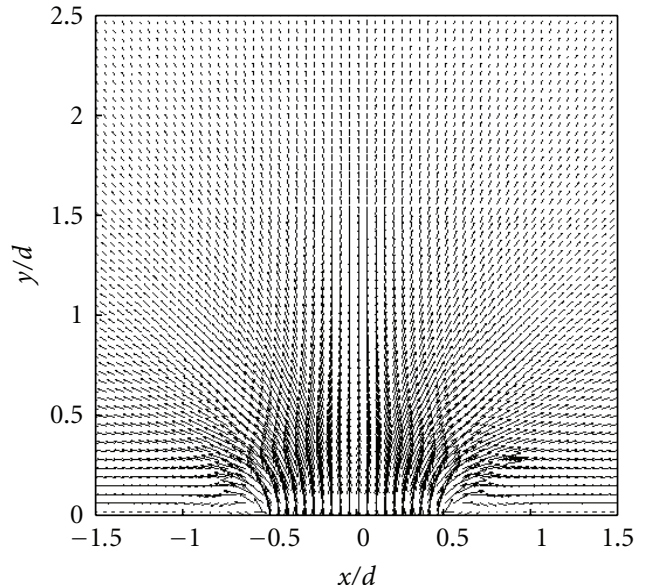

(b)

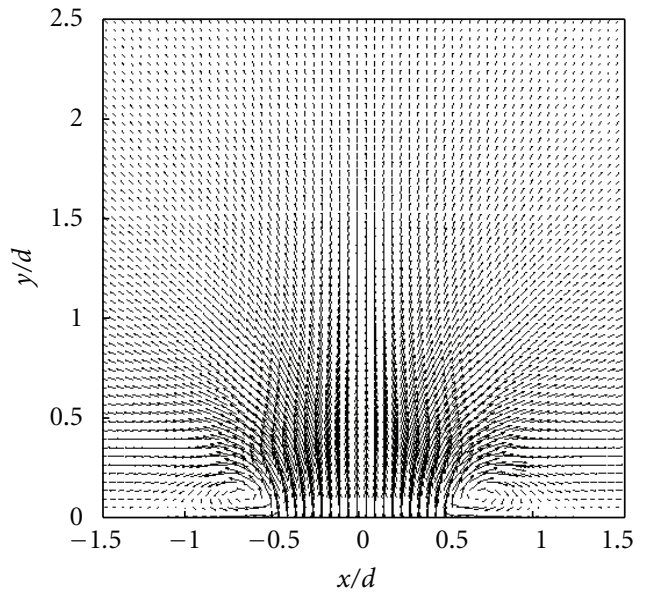

(d)

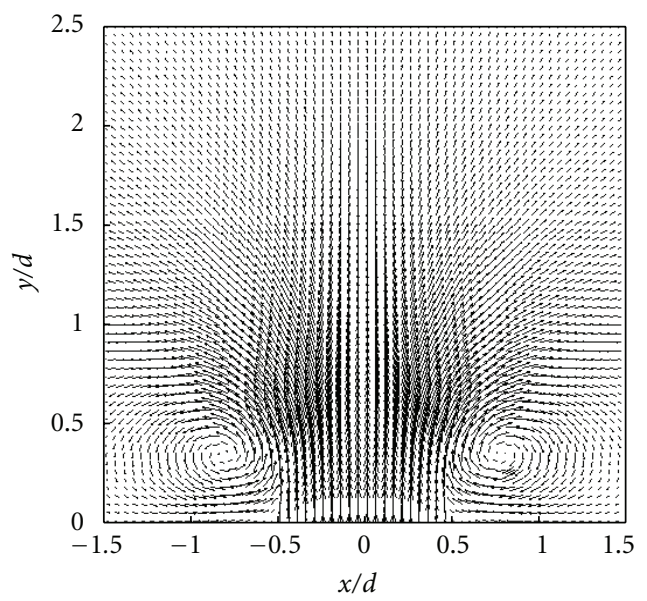

(f)

FIGURE 5: Comparison of experimental and numerical velocity vector plots during four phases of expulsion phase: $t=0.14((\mathrm{a})$, (b)), $t=0.18((\mathrm{c}),(\mathrm{d}))$, and $t=0.26((\mathrm{e}),(\mathrm{f}))$. 


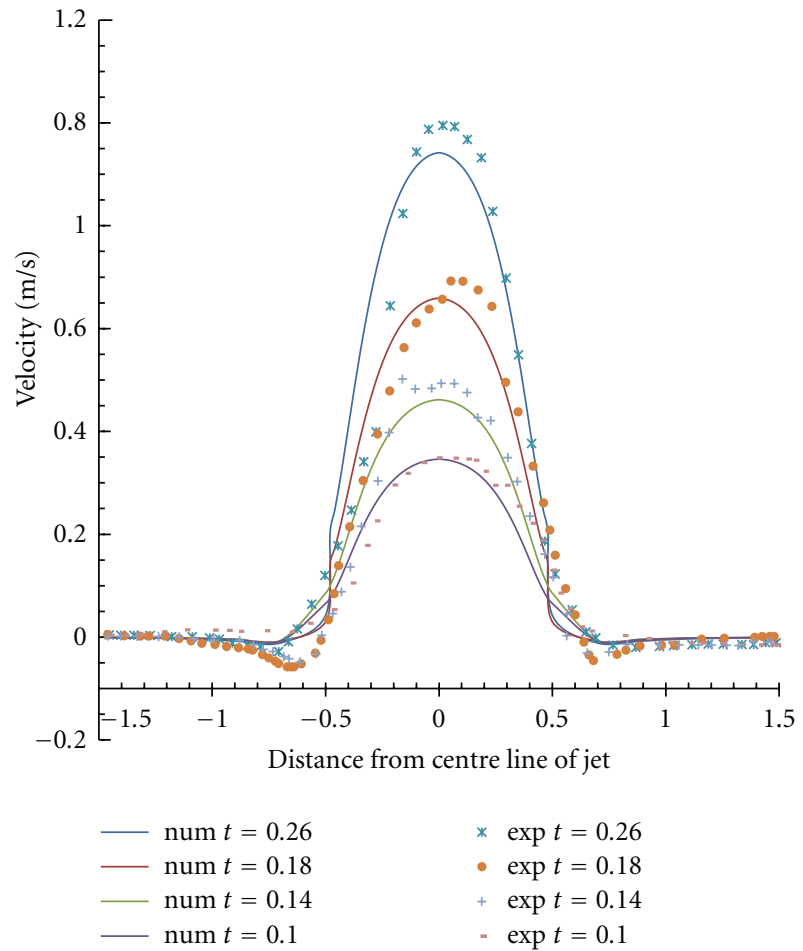

Figure 6: Comparison of experimental and numerical streamwise component of velocity profiles at orifice exit during the four phases of the expulsion phase.

the first half of the expulsions stage, that is, at $t=0.26$, two apparent vortices are seen at about half orifice diameter away from the orifice exit. The vector plots presented show good agreement in terms of vortex core location and general flow field for numerical and experimental results. A slight asymmetry in the experimental results can be seen in all three vector plots. The vortex core on the right side of the plots appears further downstream than the vortex core on the left side at each phase. Possible reason could be attributed to slight imperfections at the test section orifice or a misalignment between the desired test section plane and the plane of focus of the microscope lens.

As for quantitative data comparison, the row of velocity vectors located closest to the plane of the orifice, $0.1 \mathrm{~d}$ in front, has been used to extract the orifice exit profiles of streamwise velocity components. In Figure 6, profiles from the first half of the expulsion part of the cycle are presented. The velocity distribution early in the expulsion phase, $t=0.1$, showed a developing profile which becomes parabolic by the time it reaches its maximum, $t=0.26$. At this time, numerical solution recorded a maximum velocity of $0.979 \mathrm{~m} / \mathrm{s}$ in the near vicinity of the orifice, whereas experimental data recorded a value of $0.985 \mathrm{~m} / \mathrm{s}$. Overall, both numerical and experimental results showed good agreement for all of the time instants. Again, experimental results show the asymmetry velocity profile as the peak velocity did not reside on the centreline.

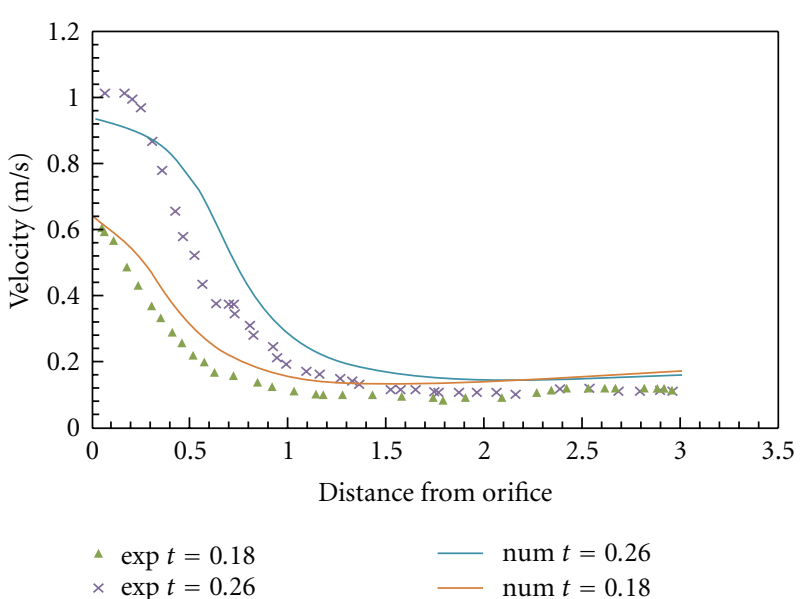

(a)

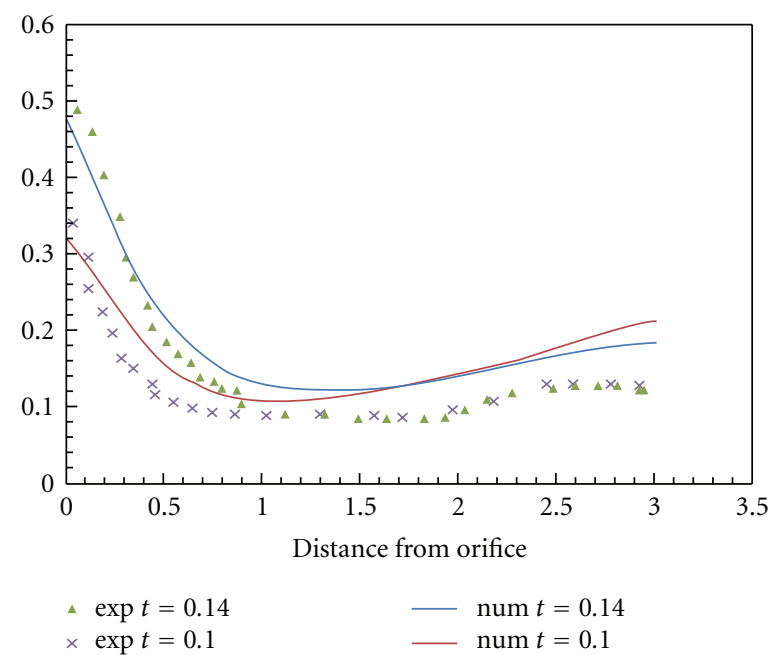

(b)

Figure 7: Comparison of experimental and numerical streamwise component of velocity profiles along the jet centreline during the four phases of the expulsion phase.

In Figure 7 the streamwise component of velocity along the centreline was compared for the same part of the expulsion phase. The small discrepancies of velocity magnitude could be due to the misalignment of the test device as spotted in the asymmetry velocity profile in Figure 6 which then resulted in slightly higher centreline velocity right above orifice. Overall, Figure 7 shows good agreement of numerical results in predicting the general trend of velocity profiles at orifice exit and along the centreline of the pulsating jet for all times. This leads us to the conclusion that the phase locations determined for the experimental results from the numerical data based on peak velocity were reasonable.

\section{Conclusions}

The moving mesh algorithm as proposed was found to be efficient and accurate as it eradicated the need for 
either re-meshing or ssolving a new evolution equation for the Jacobian. This method also enforced GCL as grid points were moved by using mesh velocities and not the other way round. The comparison presented demonstrates good agreement, thereby confirming that the moving mesh algorithm developed is valid and is able to resolve the flow fields of microsynthetic jets. The validity of the moving mesh algorithm was assessed against the measured streamwise component of velocity profiles at the orifice exit and along the centerline of the pulsating jet in a micro-channel as well as the location of vortex core at different time instants of the expulsion stage. Good agreement was achieved between the measurements and numerical predictions. The comparison presented thereby confirms that the moving mesh algorithm is developed to be valid and able to resolve the flow fields of micro synthetic jets.

\section{Nomenclature}

$\begin{array}{ll}d: & \text { Orifice diameter } \\ f: & \text { Actuator frequency } \\ f(d): & \text { Weighting function } \\ h_{l}: & \text { Fluid enthalpy } \\ H: & \text { Depth } \\ \sqrt{g}: & \text { Jacobian of body-fitted coordinate system } \\ L: & \text { Length } \\ n: & \text { Outward drawn normal } \\ p: & \text { Fluid pressure } \\ P^{i}, P^{j} P^{k}: \text { Interpolation or "blending" functions } \\ t: & \text { Time in the physical coordinates } \\ t^{*}: & \text { Time, } s \\ T: & \text { Period of actuation cycle } \\ U: & \text { Fluid velocity } \\ U_{g}: & \text { Grid velocity } \\ W: & \text { Width } \\ w: & \text { Width of the micro-channel } \\ Y_{m}: & \text { Displacement of membrane } \\ x, y, z: & \text { Physical coordinates in the Cartesian frame. }\end{array}$

\section{Greek Symbols}

$\beta_{i l}, \beta_{l m}$ : Grid transformation coefficients

$\rho_{l}: \quad$ Fluid density

$\mu_{l}: \quad$ Fluid viscosity

$\tau$ : $\quad$ Time in the computational coordinates

$\tau^{\prime}$ : $\quad$ Period of oscillation

$\varepsilon, \gamma, \omega$ : Body-fitted coordinate system.

\section{References}

[1] A. Glezer and M. Amitay, "Synthetic jets," Annual Review of Fluid Mechanics, vol. 34, pp. 503-529, 2002.

[2] M. Gordon and J. Soria, "Scalar mixing of zero-net-mass-flux jets in crossflow," in Proceedings of the 14th Australasian Fluid Mechanics Conference, pp. 729-732, 2001.

[3] J. S. Campbell, W. Z. Black, A. Glezer, and J. G. Hartley, "Thermal management of a laptop computer with synthetic air microjets," in Proceedings of the 6th Intersociety Conference on Thermal and Thermomechanical Phenomena in Electronic Systems (ITHERM '98), pp. 43-50, May 1998.

[4] R. Mahalingam and A. Glezer, "Air cooled heat sinks integrated with synthetic jets," in Proceedings of the 8th Intersociety Conference on Thermal and Thermommechanical Phenomena in Electronic Systems, pp. 285-291, June 2002.

[5] R. Mahalingam, N. Rumigny, and A. Glezer, "Thermal management using synthetic jet ejectors," IEEE Transactions on Components and Packaging Technologies, vol. 27, no. 3, pp. 439-444, 2004.

[6] B. L. Smith and G. W. Swift, "A comparison between synthetic jets and continuous jets," Experiments in Fluids, vol. 34, no. 4, pp. 467-472, 2003.

[7] A. Pavlova and M. Amitay, "Electronic cooling using synthetic jet impingement," Journal of Heat Transfer, vol. 128, no. 9, pp. 897-907, 2006.

[8] M. B. Gillespie, W. Z. Black, C. Rinehart, and A. Glezer, "Local convective heat transfer from a constant heat flux flat plate cooled by synthetic air jets," Journal of Heat Transfer, vol. 128, no. 10, pp. 990-1000, 2006.

[9] Z. Trávníček and V. Tesař, "Annular synthetic jet used for impinging flow mass-transfer," International Journal of Heat and Mass Transfer, vol. 46, no. 17, pp. 3291-3297, 2003.

[10] Z. Trávníček and V. Tesař, "Annular impinging jet with recirculation zone expanded by acoustic excitation," International Journal of Heat and Mass Transfer, vol. 47, no. 10-11, pp. 23292341, 2004.

[11] J. Lepičovský, K. K. Ahuja, and R. H. Burrin, "Tone excited jets, part III: flow measurements," Journal of Sound and Vibration, vol. 102, no. 1, pp. 71-91, 1985.

[12] J. Lepičovský, K. K. Ahuja, and M. Salikuddin, "An experimental study of tone-excited heated jets," Journal of Propulsion and Power, vol. 2, no. 2, pp. 149-154, 1986.

[13] W. J. Gordon and L. C. Thiel, "Transfinite mappings and their application to grid generation," in Numerical Grid Generation, J. F. Thompson, Ed., pp. 171-192, Elsevier, New York, NY, USA, 1982.

[14] S. F. Anwer, N. Hasan, S. Sanghi, and S. Mukherjee, "Computation of unsteady flows with moving boundaries using body fitted curvilinear moving grids," Computers and Structures, vol. 87, no. 11-12, pp. 691-700, 2009.

[15] C. M. Rhie and W. L. Chow, "An numerical study of the turbulent flow past an airfoil with trailing edge separation," AIAA Journal, vol. 21, no. 11, pp. 1525-1532, 1983.

[16] H. L. Stone, "Iterative solution of implicit approximations of multidimensional partial differential equations," SIAM Journal on Numerical Analysis, vol. 5, pp. 530-558, 1968.

[17] A. Sinclair, V. Timchenko, J. A. Reizes, G. Rosengarten, and E. Leonarid, "An experimental and numerical study of a micro-synthetic jet in a shallow cavity," in Proceedings of the 6th ASME Conference on Nanochannels, Microchannels and Minichannels, Darmstadt, Germany, 2008.

[18] K. Haubert, T. Drier, and D. Beebe, "PDMS bonding by means of a portable, low-cost corona system," Lab on a Chip, vol. 6, no. 12, pp. 1548-1549, 2006. 

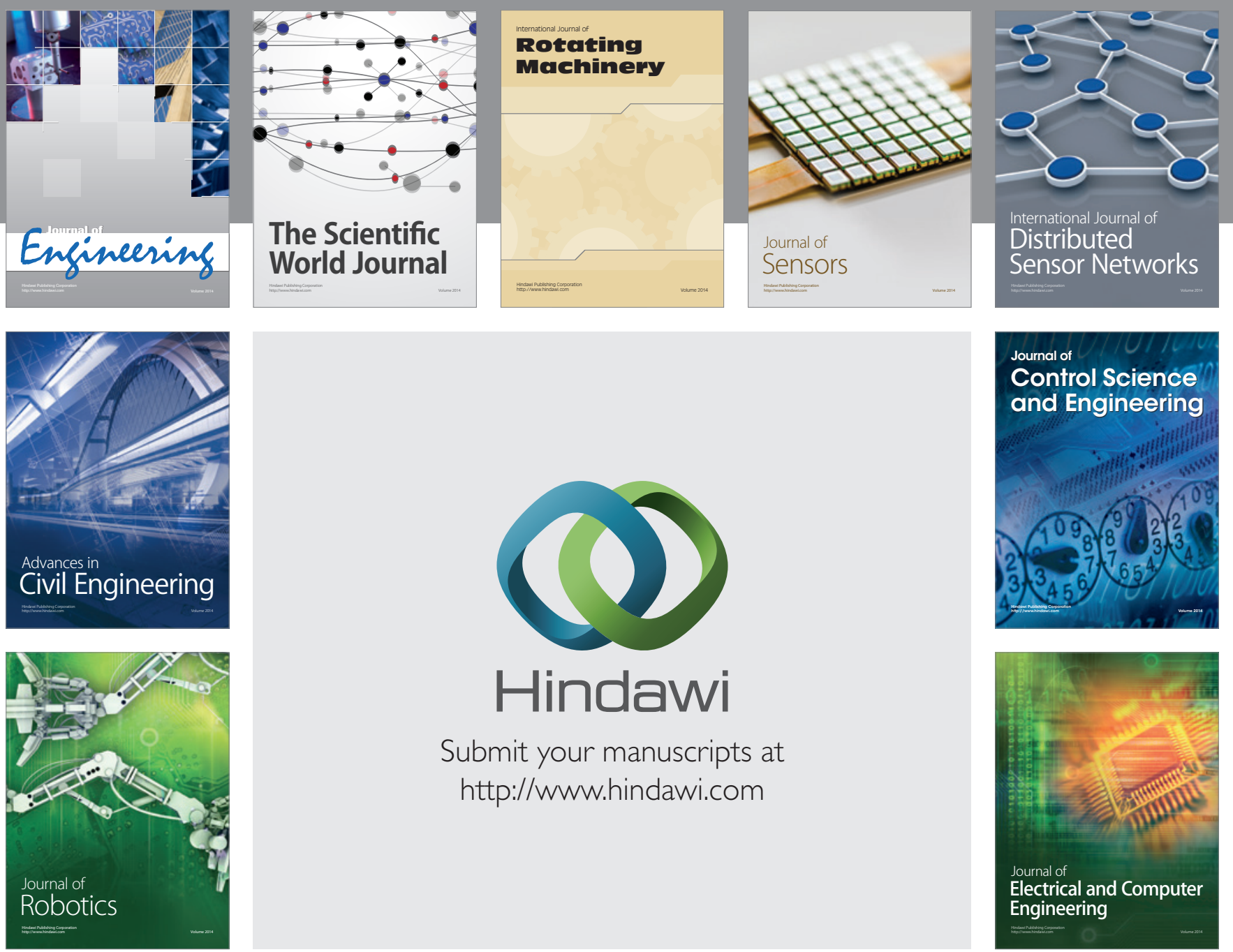

Submit your manuscripts at

http://www.hindawi.com
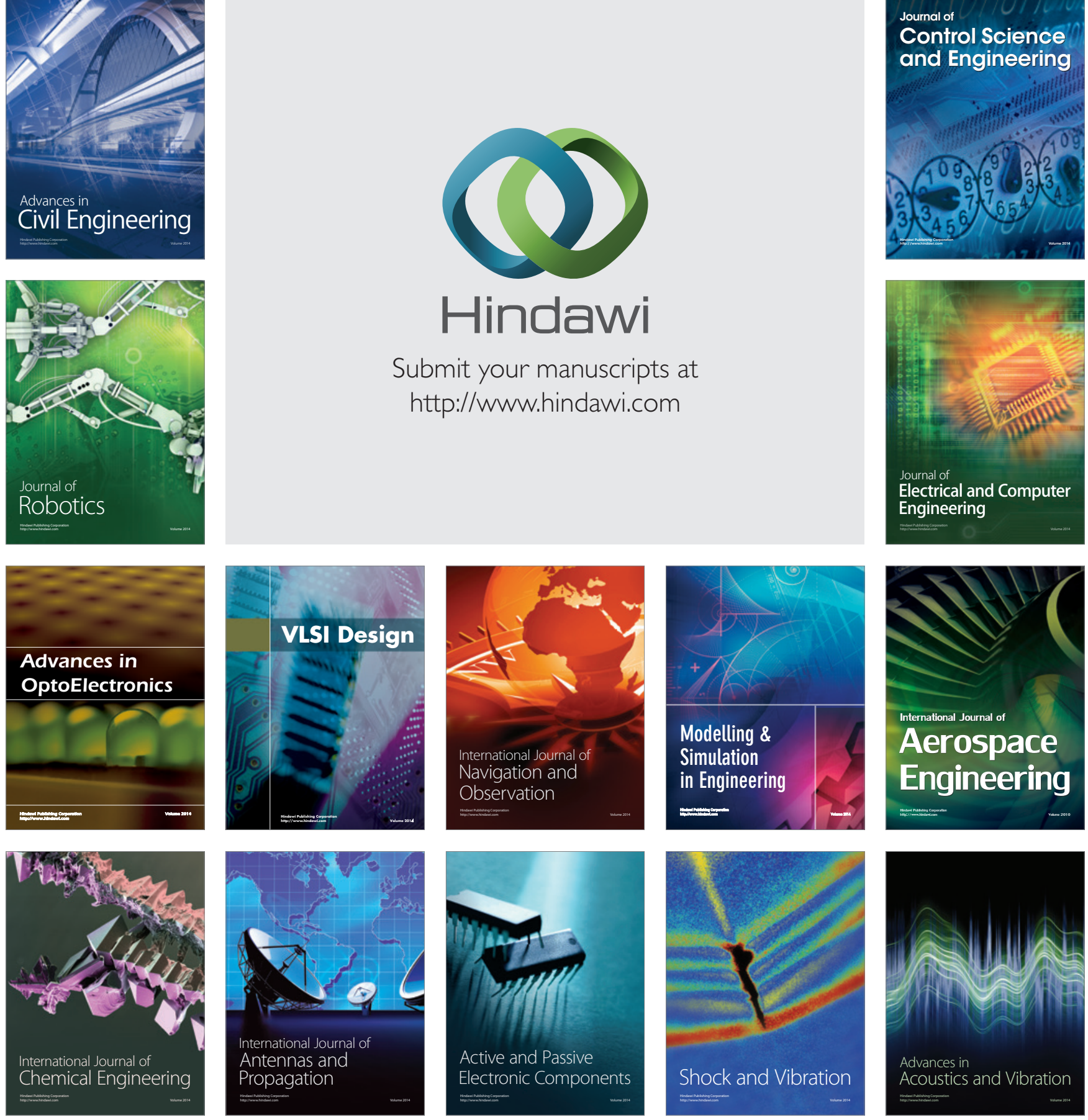